\title{
Association between polymorphisms in the estrogen receptor alpha gene and osteoarthritis susceptibility: a meta-analysis
}

Yan Ren ${ }^{1 \dagger}$, Bo Tann ${ }^{2 \dagger}$, Peijing Yan ${ }^{1}$, Yi You ${ }^{3}$, Yanqiao $\mathrm{Wu}^{1,4^{*}}$ and Yue Wang ${ }^{2^{*}}$

\begin{abstract}
Background: Osteoarthritis $(\mathrm{OA})$ is a common chronic disease of the joints. Genetic factors may play a role in its development, and polymorphisms in the estrogen receptor alpha gene (ERa) have been associated with OA. However, previous studies into this relationship have reported inconsistent results, so we aimed to systematically review the association between ERa polymorphisms and OA susceptibility.

Methods: We conducted a comprehensive literature search of Ovid MEDLINE, EMBASE, CBM, and PubMed databases, and Google scholar, and identified 11 eligible studies that examined the association between ERa polymorphisms and OA susceptibility. We carried out a meta-analysis of these studies based on ERa Xbal (rs9340799) and Pvull (rs2234693) genotypes.
\end{abstract}

Results: Seventeen comparisons involving 10 European and seven Asian populations of 5,325 OA patients and 10,834 controls were included in the study. The ERa Xbal polymorphism were significantly associated with OA in Europeans (AA vs. $A G+G G: O R=1.17,95 \%$ confidence interval $(C l)=1.02-1.34, P=0.03 ; A G$ vs. $A A+G G: O R=0.86,95 \% C l=0.75-0.99$, $P=0.04$ ) but not in Asian populations. No association was found between $O A$ and the ERa Pvull polymorphism in any population ( $C$ vs. $T, O R=0.98,95 \% C l=0.93-1.03, P=0.37 ; C C$ vs. $T T+C T, O R=0.97,95 \% C l=0.89-1.06, P=0.55 ; C T$ vs. $C C+\pi, O R=0.99,95 \% C l=0.92-1.06, P=0.75 ; \pi$ vs. $C C+C T, O R=1.01,95 \% C l=0.92-1.12, P=0.79)$.

Conclusions: This study suggested that there may be a weak relationship between the ERa Xbal polymorphism and OA in Europeans but not Asians, and that the ERa Pvull polymorphism was not associated with OA in either population. However, large well-designed studies are necessary to confirm these results in more homogeneous populations.

Keywords: Estrogen receptor, Osteoarthritis, Polymorphism, Meta-analysis

\section{Background}

Osteoarthritis (OA) is the most common joint disease worldwide, and primarily affects the knees, hips, hands, and spine. It is a leading cause of disability among older individuals and also affects their quality of life [1]. It is characterized by the progressive degeneration of articular cartilage, and by subchondral sclerosis resulting in pain and joint stiffness [2].

\footnotetext{
*Correspondence: wu_yanqiao@163.com; 283546806@qq.com

${ }^{\dagger}$ Equal contributors

${ }^{1}$ Department of Epidemiology and Biostatistics, West China School of Public Health, Sichuan University, Chengdu 610041, People's Republic of China ${ }^{2}$ Department of Orthopedics, Sichuan Academy of Medical Sciences, Sichuan Provincial People's Hospital, Chengdu 610072, People's Republic of China Full list of author information is available at the end of the article
}

The etiology of OA is multifactorial, including genetic and environmental risk factors. Associated genes include GDF5 [3], ASPN [4], FRZB [5], and COL2A1 [6], while environmental factors may include obesity [7-9], history of knee injury [10], occupational activities [11,12], sex hormones and structural changes [13], meniscectomy [14], gender, and age [15]. Twin-pair and family genetic data show that more than $50 \%$ of OA can be attributed to genetic factors [16]. A gender difference is also apparent, with females having a greater prevalence of OA after the age of 50 years [17]. Additionally, the disease is more common among European populations [18]. The observation that the estrogen receptor (ER) is expressed in human articular chondrocytes and bone cells suggests that it may be involved in the etiology of OA [19]. 
The ER has two isoforms: ER $\alpha$ and ER $\beta . E R \alpha$ expression affects the growth of bone cells, while $E R \beta$ participates in the formation and resorption of bone [20]. ER $\alpha$ is located on chromosome 6q25.1 and contains eight exons and seven introns [21], as well as two common restriction fragment length polymorphisms (RFLPs): XbaI and PvuII. The $X b a \mathrm{I}$ RFLP detects an A-G substitution at position 351 (-351int A/G; rs9340799), while PvuII detects a T-C substitution at position 397 (-397int T/C; rs2234693). A previous meta-analysis confirmed the association between bone mineral density and ER $\alpha$ [22].

A number of studies have investigated the association between $E R \alpha$ polymorphisms and the risk of OA in different populations, but the results are inconsistent. Some discovered that ER $\alpha$ polymorphisms were associated with an increased risk of OA [23-28], while others found no association with OA risk $[28,29]$, or an association with a reduced risk of OA [30-34]. To our knowledge, no systematic review has examined the evidence for a relationship between $E R \alpha$ polymorphisms and OA. Therefore, we conducted a meta-analysis to analyze the association between $E R \alpha$ polymorphisms and OA susceptibility.

\section{Methods}

This systematic review was conducted according to 2009 PRISMA guidelines [35].

\section{Search strategy}

We performed a systematic research of available studies that assessed the association between ER $\alpha$ polymorphisms and OA. We carried out a comprehensive literature search for published studies in OVID MEDLINE, EMBASE, CBM, and PubMed databases, and Google Scholar. Primary key search terms included estrogen receptor, polymorphism, osteoarthritis, and OA. Index terms for OVID MEDLINE were: "estrogen receptor", "polymorphism", and "osteoarthritis" or "OA". The last query was updated on 30 November 2014. There were no language or other limitations on the search. Reference lists in the retrieved articles or relevant reviews were also screened to identify other eligible studies. We also searched unpublished studies by contacting clinical experts and the Arthritis Foundation National Office. A flow diagram of our literature identification strategy is shown in Figure 1.

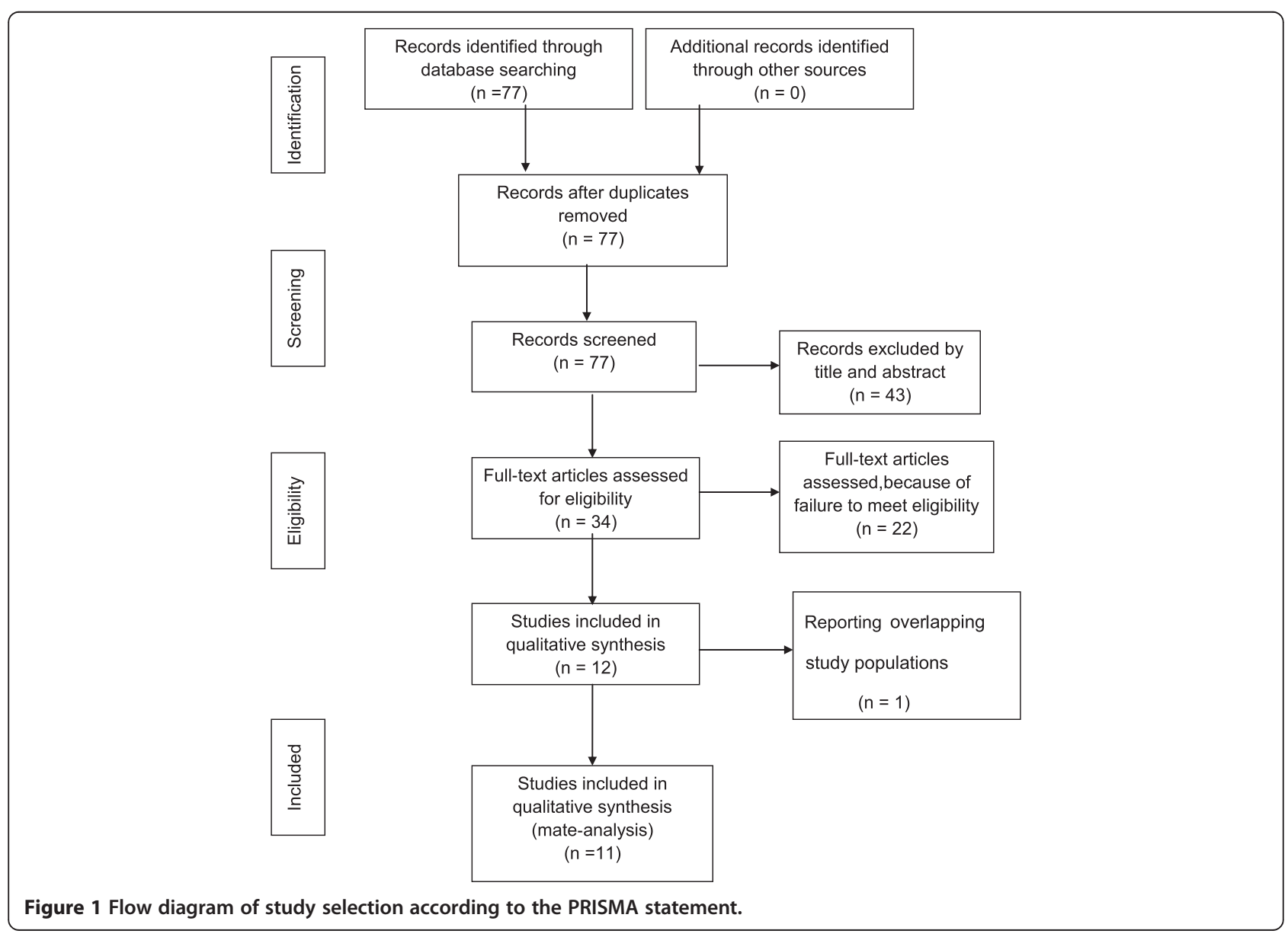




\section{Inclusion and exclusion criteria}

Eligible studies were required to satisfy the following criteria: (1) the study was a cohort or a case-control study; (2) OA was diagnosed based on clinical criteria defined by the American College of Rheumatology; (3) the original study assessed the association between ER $\alpha$ polymorphisms (XbaI or PvuII) and OA susceptibility; and (4) the study provided sufficient genetic frequency or sufficient data for extraction. If overlapping study populations were identified between studies, only the most complete one was included in the meta-analysis. Animal studies and literature reviews were excluded.

\section{Quality assessment of included studies}

Study quality was independently assessed by two authors, based on the Newcastle-Ottawa scale (NOS) quality score systems [36]. The NOS contains eight items divided into three categories: selection, comparability, and outcome (for cohort studies) or exposure (for case-control studies). Quality scores ranged from 0 to 9 . When there was disagreement on the quality scores between the two authors, discrepancies were resolved through discussion and consultation with a third author.

The quality of included studies was also assessed by the Hardy-Weinberg equilibrium (HWE) for the control genotype distribution. Studies consistent with HWE were defined as high-quality, while those inconsistent with HWE were defined as low-quality studies.

\section{Data extraction}

The following data were extracted from each full-text study using a standardized data extraction form: the name of the first author, year of publication, country in which the study was performed, study design, number of cases and controls, gender, age, genotyping, OA site, OA definition, polymorphism, and numbers of cases and controls for each of the PvuII (rs2234693), and XbaI (rs9340799) genotypes. When the information extracted from studies was inconsistent, disagreement was resolved through discussion and consultation with a third author until consensus was achieved on every item.

\section{Statistical analysis}

STATA 12.0 and Review Manager 5.2 software were used for data analysis. The pooled odds ratio (OR) and its $95 \%$ confidence interval ( $95 \% \mathrm{CI}$ ) were calculated to assess the association between $E R \alpha$ polymorphisms and the risk of $\mathrm{OA}$ for the following contrasts: $G$ vs. A, AG vs. $A A+G G, G G$ vs. $A G+A A, A A$ vs. $A G+G G, C$ vs. $\mathrm{T}, \mathrm{CC}$ vs. TT + CT, CT vs. $\mathrm{CC}+\mathrm{TT}$, and TT vs. $\mathrm{CC}+$ CT. Subgroup analysis based on ethnicity was also performed. The Chi-square test was used to determine if the identified study was consistent with HWE for the control genotype distribution. Heterogeneity between studies was evaluated with the $I^{2}$ test and the $Q$ statistic. We used the Cochrane system for heterogeneity grading: $I^{2}$ 0-40\%, might not be important; 30-60\%, moderate heterogeneity; $50-90 \%$, substantial heterogeneity; $75-100 \%$, considerable heterogeneity. Heterogeneity was assessed to be significant when $I^{2}>30 \%$ or when $P<0.1$ for $Q$ statistics.

The pooled effects were estimated using the DerSimonian and Laird method for random effects and the Mantel-Haenszel method for fixed effects [37]. If the studies were significantly heterogeneous, we used the random effects model. Otherwise, we used the fixed effects model to calculate the pooled OR and $95 \% \mathrm{CI}$. The random effects model assumes that different studies have substantial diversity and assesses both within-study sampling error and between-study variation [38]. The fixed effects model assumes that genetic factors have similar effects on OA susceptibility across all studies, and that observed variations between studies are caused by chance alone [39]. Sensitivity analyses were performed for the effect size omitting the trial for which data were imputed, and were used to evaluate the stability of the results. Publication bias was graphically represented by funnel plots and further evaluated with the Begg's test and Egger's test $[40,41]$.

\section{Results}

Search results and studies included in the meta-analysis Seventy-seven relevant studies were preliminarily identified in the database search, of which 11 [24-34] eventually satisfied the eligibility criteria for our meta-analysis. All included studies investigated the relationship between $E R \alpha$ polymorphisms and OA susceptibility. Of these, one study [33] contained data on three different OA sites and four different geographical locations, so these seven comparisons were treated independently. Therefore, a total of 17 separate comparisons were included in the present meta-analysis. Ten studies with a total of 8,502 participants (2,181 OA patients and 6,321 controls), which involved three European and seven Asian populations, evaluated the association between the ER $\alpha \mathrm{XbaI}$ polymorphism and OA susceptibility, while 17 with 16,159 total participants (5,325 OA patients and 10,834 controls), involving 10 European and seven Asian populations, evaluated the association between the ER $\alpha$ PvuII polymorphism and OA susceptibility. Study characteristics are summarized in Table 1.

\section{Allele and genotype counts}

Allelic counts of the ER $\alpha b a \mathrm{I}$ polymorphism were evaluated for $\mathrm{G}$ and $\mathrm{A}$ alleles. In general, the frequency of the A allele was higher in OA cases than in controls. Genotype counts of the ER $\mathrm{XbaI}$ polymorphism were evaluated for GG, AG, and AA genotypes, and the 


\section{Table 1 Characteristics of the included studies}

\begin{tabular}{|c|c|c|c|c|c|c|c|c|c|c|c|c|}
\hline \multirow[t]{2}{*}{ Study [Ref.] } & \multirow[t]{2}{*}{ Year } & \multirow[t]{2}{*}{ Country (City) } & \multirow{2}{*}{$\begin{array}{l}\text { Study } \\
\text { design }\end{array}$} & \multirow[t]{2}{*}{ Genotyping } & \multicolumn{2}{|c|}{ Numbers } & \multicolumn{2}{|c|}{ Gender (M/F) } & \multicolumn{2}{|l|}{ Age } & \multirow[t]{2}{*}{ Polymorphism (s) } & \multirow{2}{*}{$\begin{array}{l}\text { Quality } \\
\text { score }\end{array}$} \\
\hline & & & & & OA & Control & OA & Control & $\mathrm{OA}$ & Control & & \\
\hline Toshio Ushiyama et al. [24] & 1998 & Japan & Case-control & PCR & 65 & 318 & $0 / 65$ & $0 / 318$ & $68.5(49-86)$ & $49-86$ & Xbal, Pvull & $7(2 / 2 / 3)$ \\
\hline John Loughlin et al. [29] & 2000 & UK (Oxford) & Case-control & PCR & 371 & 369 & $155 / 216$ & $221 / 148$ & $73(56-90)$ & $73(59-89)$ & Xbal, Pvull & $8(3 / 2 / 3)$ \\
\hline Barton L. Wise et al. [30] & 2009 & USA & Cohort & PCR & 307 & 214 & $258 / 263$ & & $61 \pm 9$ & & Xbal & $8(4 / 2 / 2)$ \\
\hline Barton L. Wise et al. [30] & 2009 & USA & Cohort & PCR & 304 & 211 & $253 / 262$ & & $61 \pm 9$ & & Pvull & \\
\hline V. M. Borgonio-Cuadra et al. [32] & 2012 & Mexico & Case-control & PCR & 115 & 117 & 23/92 & 20/97 & $57.4 \pm 9.2$ & $51.8 \pm 8.9$ & Xbal, Pvull & $9(4 / 2 / 3)$ \\
\hline J. A. Riancho et al. [33] & 2010 & Spain (Santander) & Case-control & PCR & 272 & 802 & $95 / 177$ & $285 / 517$ & $72 \pm 7$ & $71 \pm 10$ & Pvull & $8(3 / 2 / 3)$ \\
\hline J. A. Riancho et al. [33] & 2010 & Spain (Santiago) & Case-control & PCR & 254 & 473 & $47 / 207$ & 295/178 & $68 \pm 6$ & $68 \pm 9$ & Pvull & \\
\hline J. A. Riancho et al. [33] & 2010 & UK (Oxford) & Case-control & PCR & 445 & 862 & $176 / 269$ & $471 / 391$ & $64 \pm 5$ & $69 \pm 7$ & Pvull & \\
\hline J. A. Riancho et al. [33] & 2010 & Spain (Santander) & Case-control & PCR & 359 & 802 & 180/179 & $285 / 517$ & $71 \pm 7$ & $71 \pm 10$ & Pvull & \\
\hline J. A. Riancho et al. [33] & 2010 & Spain (Coruña) & Case-control & PCR & 252 & 244 & $90 / 162$ & $97 / 147$ & $67 \pm 14$ & $65 \pm 13$ & Pvull & \\
\hline J. A. Riancho et al. [33] & 2010 & Spain (Santiago) & Case-control & PCR & 287 & 473 & 110/177 & $295 / 178$ & $68 \pm 5$ & $68 \pm 9$ & Pvull & \\
\hline J. A. Riancho et al. [33] & 2010 & UK (Oxford) & Case-control & PCR & 1278 & 862 & $503 / 775$ & $471 / 391$ & $65 \pm 6$ & $69 \pm 7$ & Pvull & \\
\hline K. Lian M.D. et al. [31] & 2007 & USA & Cohort & PCR & 569 & 4134 & $0 / 569$ & 0/4134 & $79.6 \pm 5.0$ & $78.4 \pm 4.6$ & Xbal, Pvull & $8(4 / 2 / 2)$ \\
\hline Sheng-Yu Jin et al. [25] & 2004 & Korea & Case-control & PCR & 151 & 397 & $53 / 98$ & 190/207 & $58.8 \pm 9.6$ & / & Xbal, Pvull & $8(3 / 2 / 3)$ \\
\hline Zhi Tian et al. [28] & 2009 & China & Case-control & PCR & 38 & 40 & $0 / 38$ & $0 / 40$ & $59.2 \pm 3.2$ & $58.5 \pm 8.6$ & Xbal, Pvull & $7(2 / 2 / 3)$ \\
\hline Jiexiang Yang et al. [34] & 2009 & China & Case-control & PCR & 41 & 40 & $31 / 50$ & & $54.6(28-82)$ & & Xbal, Pvull & $6(2 / 1 / 3)$ \\
\hline Yan Xue et al. [27] & 2004 & China & Case-control & PCR & 55 & 176 & $0 / 55$ & 0/176 & $58.7 \pm 2.4$ & $60 \pm 10$ & Xbal, Pvull & $7(3 / 1 / 3)$ \\
\hline Xiaoyu Dai et al. [26] & 2014 & China & Case-control & $P C R$ & 469 & 522 & $113 / 356$ & $398 / 124$ & $57.3 \pm 10.9$ & $56.4 \pm 9.8$ & Xbal, Pvull & $7(3 / 1 / 3)$ \\
\hline
\end{tabular}


Table 2 Genotype and allele counts for the ERa Xbal polymorphism in the included studies

\begin{tabular}{|c|c|c|c|c|c|c|c|c|c|c|c|c|c|}
\hline \multirow[t]{2}{*}{ Group } & \multirow[t]{2}{*}{ Study } & \multirow[t]{2}{*}{ Country } & \multirow[t]{2}{*}{ OA site } & \multicolumn{2}{|l|}{$X(G)$} & \multicolumn{2}{|l|}{$x(A)$} & \multicolumn{2}{|c|}{$x x(A A)$} & \multicolumn{2}{|c|}{$X x(A G)$} & \multicolumn{2}{|c|}{$X X(G G)$} \\
\hline & & & & $\mathrm{OA}$ & Control & $\overline{O A}$ & Control & $\overline{O A}$ & Control & $\mathrm{OA}$ & Control & $O A$ & Control \\
\hline \multirow[t]{3}{*}{ European } & Barton L. Wise et al. & USA & Hand & 202 & 159 & 412 & 269 & 148 & 85 & 116 & 99 & 43 & 30 \\
\hline & John Loughlin et al. & UK & Hip,Knee & 256 & 251 & 486 & 487 & 164 & 161 & 158 & 165 & 49 & 43 \\
\hline & K. Lian M.D. et al. & USA & Hip & 374 & 2914 & 764 & 5332 & 257 & 1700 & 250 & 1932 & 62 & 491 \\
\hline European Total & & & & 832 & 3324 & 1662 & 6088 & 569 & 1946 & 524 & 2196 & 154 & 564 \\
\hline \multirow[t]{7}{*}{ Asian } & Sheng-Yu Jin et al. & Korea & Knee & 57 & 156 & 245 & 638 & 98 & 256 & 49 & 126 & 4 & 15 \\
\hline & Toshio Ushiyama et al. & Japan & Hand & 30 & 116 & 100 & 520 & 36 & 211 & 28 & 98 & 1 & 9 \\
\hline & V. M. Borgonio-Cuadra et al. & Mexico & Knee & 49 & 63 & 181 & 171 & 70 & 62 & 41 & 47 & 4 & 8 \\
\hline & Zhi Tian et al. & China & Knee & 24 & 47 & 52 & 33 & 18 & 6 & 16 & 21 & 4 & 13 \\
\hline & Jiexiang Yang et al. & China & Knee & 15 & 19 & 67 & 61 & 28 & 24 & 11 & 13 & 2 & 3 \\
\hline & Yan Xue et al. & China & Knee & 44 & 200 & 66 & 162 & 21 & 40 & 24 & 82 & 10 & 54 \\
\hline & Xiaoyu Dai et al. & China & Knee & 210 & 193 & 728 & 851 & 288 & 348 & 152 & 155 & 29 & 19 \\
\hline Asian Total & & & & 429 & 794 & 1439 & 2436 & 559 & 947 & 321 & 542 & 54 & 121 \\
\hline Total & & & & 1261 & 4118 & 3101 & 8524 & 1128 & 2893 & 845 & 2738 & 208 & 685 \\
\hline
\end{tabular}

frequency of the AA genotype was higher in OA cases than in the control group in all but one study [24]. The frequency of the AG genotype was lower in OA cases than in the control group in all but the same study [24]. There was no obvious difference in the frequency of the
GG genotype between OA cases and controls. Allele and genotype counts for the $E R \alpha X b a \mathrm{I}$ polymorphism in cases and controls are shown in Table 2.

Allelic counts of the ER $\alpha$ PvuII polymorphism were evaluated for $\mathrm{C}$ and $\mathrm{T}$ alleles. In general the $\mathrm{T}$ allele frequency

Table 3 Genotype and allele counts for the ERa Pvull polymorphism in the included studies

\begin{tabular}{|c|c|c|c|c|c|c|c|c|c|c|c|c|c|}
\hline \multirow[t]{2}{*}{ Group } & \multirow[t]{2}{*}{ Study } & \multirow[t]{2}{*}{ Coutry (City) } & \multirow[t]{2}{*}{ OA site } & \multicolumn{2}{|l|}{$P(C)$} & \multicolumn{2}{|l|}{$p(T)$} & \multicolumn{2}{|c|}{ pp (TT) } & \multicolumn{2}{|c|}{$\mathrm{Pp}(\mathrm{CT})$} & \multicolumn{2}{|c|}{$\mathrm{PP}(\mathrm{CC})$} \\
\hline & & & & $\overline{O A}$ & $\overline{\text { Control }}$ & $\overline{O A}$ & Control & $\overline{O A}$ & $\overline{\text { Control }}$ & $\overline{O A}$ & $\overline{\text { Control }}$ & $\overline{O A}$ & Control \\
\hline \multirow[t]{10}{*}{ European } & Barton L. Wise et al. & USA & Hand & 261 & 192 & 347 & 230 & 101 & 65 & 145 & 100 & 58 & 46 \\
\hline & J. A. Riancho et al. & Spain (Coruña) & Hip & 213 & 217 & 291 & 271 & 89 & 76 & 113 & 119 & 50 & 49 \\
\hline & J. A. Riancho et al. & UK (Oxford) & Hip & 1109 & 776 & 1447 & 948 & 426 & 253 & 595 & 442 & 257 & 167 \\
\hline & J. A. Riancho et al. & UK (Oxford) & Knee & 399 & 776 & 491 & 948 & 123 & 253 & 245 & 442 & 77 & 167 \\
\hline & J. A. Riancho et al. & Spain (Santander) & Hip & 334 & 752 & 384 & 852 & 105 & 229 & 174 & 394 & 80 & 179 \\
\hline & J. A. Riancho et al. & Spain (Santander) & Knee & 246 & 752 & 298 & 852 & 79 & 229 & 140 & 394 & 53 & 179 \\
\hline & J. A. Riancho et al. & Spain (Santiago) & Knee & 235 & 377 & 273 & 569 & 65 & 176 & 143 & 217 & 46 & 80 \\
\hline & J. A. Riancho et al. & Spain (Santiago) & Hip & 239 & 377 & 335 & 569 & 99 & 176 & 137 & 217 & 51 & 80 \\
\hline & John Loughlin et al. & UK & Hip,Knee & 331 & 331 & 411 & 407 & 114 & 110 & 183 & 187 & 74 & 72 \\
\hline & K. Lian M.D et al. & USA & Hip & 481 & 3835 & 653 & 4391 & 188 & 1162 & 277 & 2067 & 102 & 884 \\
\hline $\begin{array}{l}\text { European } \\
\text { Total }\end{array}$ & & & & 3848 & 8385 & 4930 & 10037 & 1389 & 2729 & 2152 & 4579 & 848 & 1903 \\
\hline \multirow[t]{7}{*}{ Asian } & Sheng-Yu Jin et al. & Korea & Knee & 112 & 307 & 190 & 487 & 61 & 152 & 68 & 183 & 22 & 62 \\
\hline & Toshio Ushiyama et al. & Japan & Hand & 57 & 260 & 73 & 376 & 19 & 115 & 35 & 146 & 11 & 57 \\
\hline & $\begin{array}{l}\text { V. M. Borgonio-Cuadra } \\
\text { et al. }\end{array}$ & Mexico & Knee & 77 & 82 & 153 & 152 & 52 & 51 & 49 & 50 & 14 & 16 \\
\hline & Zhi Tian et al. & China & Knee & 29 & 34 & 47 & 46 & 16 & 15 & 15 & 16 & 7 & 9 \\
\hline & Jiexiang Yang et al. & China & Knee & 37 & 33 & 45 & 47 & 14 & 12 & 17 & 23 & 10 & 5 \\
\hline & Yan Xue et al. & China & Knee & 53 & 151 & 57 & 201 & 17 & 57 & 23 & 87 & 15 & 32 \\
\hline & Xiaoyu Dai et al. & China & Knee & 387 & 390 & 551 & 638 & 167 & 198 & 217 & 242 & 85 & 74 \\
\hline Asian Total & & & & 752 & 1257 & 1116 & 1947 & 346 & 600 & 424 & 747 & 164 & 255 \\
\hline Total & & & & 4600 & 9642 & 6046 & 11984 & 1735 & 3329 & 2576 & 5326 & 1012 & 2158 \\
\hline
\end{tabular}


was higher in OA cases than in the control group. Genotype counts of the ER $\alpha$ PvuII polymorphisms were evaluated for TT, CT, and CC genotypes, and the TT genotype frequency was generally higher in OA cases than in controls. The CC genotype frequency was generally lower in OA cases than controls, although there was no obvious difference in the frequency of the CT genotype between the two groups. Allele and genotype counts for the ER $\alpha$ $P v u I I$ polymorphism in cases and controls are shown in Table 3.

\section{Quality assessment of included studies}

All 11 studies had a satisfactory NOS quality score as shown in Table 1. The distribution of genotypes in the controls was in accordance with HWE $(P>0.05)$ in all studies, so all were classed as high-quality.

\section{Meta-analysis findings}

A summary of the meta-analysis findings are shown in Table 4. The ER $\alpha$ XbaI polymorphism was shown not to be associated with $\mathrm{OA}$ risk in all populations ( $\mathrm{G}$ vs. A: $\mathrm{OR}=0.87,95 \% \mathrm{CI}=0.73-1.04, P=0.13$; AA vs. $\mathrm{AG}+\mathrm{GG}$ : $\mathrm{OR}=1.16,95 \% \mathrm{CI}=0.94-1.44, \mathrm{P}=0.17$; $\mathrm{AG}$ vs. $\mathrm{AA}+\mathrm{GG}$ : $\mathrm{OR}=0.93,95 \% \mathrm{CI}=0.84-1.04, P=0.22$; GG vs. $\mathrm{AG}+\mathrm{AA}$ : $\mathrm{OR}=0.88,95 \% \mathrm{CI}=0.67-1.17, P=0.38$ ). However, subgroup analysis by ethnicity showed that the AA and AG genotypes of the $E R \alpha X b a \mathrm{I}$ polymorphism were associated with OA risk among Europeans (AA vs. AG + GG: OR $=1.17$, 95\% CI $=1.02-1.34, P=0.03$; AG vs. AA $+\mathrm{GG}: \mathrm{OR}=0.86$, $95 \% \mathrm{CI}=0.75-0.99, P=0.04)$, but not among Asian populations (Figure 2).

There was no significant association between the $E R \alpha$ $P v u I I$ polymorphism and susceptibility to OA in all populations ( $\mathrm{C}$ vs. $\mathrm{T}, \mathrm{OR}=0.98,95 \% \mathrm{CI}=0.93-1.03, P=0.37$; $\mathrm{CC}$ vs. $\mathrm{TT}+\mathrm{CT}, \mathrm{OR}=0.97,95 \% \mathrm{CI}=0.89-1.06, P=0.55$; CT vs. $\mathrm{CC}+\mathrm{TT}, \mathrm{OR}=0.99,95 \% \mathrm{CI}=0.92-1.06, P=0.75$; TT vs. $\mathrm{CC}+\mathrm{CT}, \mathrm{OR}=1.01,95 \% \mathrm{CI}=0.92-1.12, P=0.79$ ). In the subgroup analysis based on ethnicity, no significant association was found for the $E R \alpha P v u I I$ polymorphism in either European or Asian populations (Figure 3).

Table 4 Meta-analysis of ERa Xbal and Pvull polymorphisms and OA susceptibility

\begin{tabular}{|c|c|c|c|c|c|c|c|c|c|c|c|c|c|}
\hline \multirow{3}{*}{$\begin{array}{l}\text { Polymorphism } \\
\text { comparison }\end{array}$} & \multirow{3}{*}{$\begin{array}{l}\text { Population } \\
\text { OA site }\end{array}$} & \multirow{3}{*}{$\begin{array}{l}\text { No. of } \\
\text { studies }\end{array}$} & \multirow{2}{*}{\multicolumn{3}{|c|}{ Test of association }} & \multirow{2}{*}{\multicolumn{4}{|c|}{ Test of heterogeneity }} & \multicolumn{4}{|c|}{ Test of publication bias } \\
\hline & & & & & & & & & & \multicolumn{2}{|c|}{ Begg's test } & \multicolumn{2}{|c|}{ Egger's test } \\
\hline & & & OR & $95 \% \mathrm{Cl}$ & $p$-value & Model & Q test & $p$-value & $I^{2}$ & $\mathrm{Z}$ test & $p$-value & T test & $p$-value \\
\hline \multirow[t]{3}{*}{ Xbal (G vs. A) } & Overall & 10 & 0.87 & $0.73-1.04$ & 0.13 & Random & 29.71 & 0.0005 & $70 \%$ & -0.80 & 0.42 & -1.29 & 0.23 \\
\hline & European & 3 & 0.91 & $0.82-1.01$ & 0.08 & Fixed & 1.67 & 0.43 & $0 \%$ & & & & \\
\hline & Asian & 7 & 0.80 & $0.57-1.13$ & 0.21 & Random & 27.74 & 0.0001 & $78 \%$ & & & & \\
\hline \multirow[t]{3}{*}{$A A$ vs. $A G+G G$} & Overall & 10 & 1.16 & $0.94-1.44$ & 0.17 & Random & 25.55 & 0.002 & $65 \%$ & 1.16 & 0.25 & 0.94 & 0.38 \\
\hline & European & 3 & 1.17 & $1.02-1.34$ & 0.03 & Fixed & 1.91 & 0.39 & $0 \%$ & & & & \\
\hline & Asian & 7 & 1.22 & $0.84-1.79$ & 0.30 & Random & 21.69 & 0.001 & $72 \%$ & & & & \\
\hline \multirow[t]{3}{*}{$A G$ vs. $A A+G G$} & Overall & 10 & 0.93 & $0.84-1.04$ & 0.22 & Fixed & 10.61 & 0.30 & $15 \%$ & 0.09 & 0.93 & 0.47 & 0.65 \\
\hline & European & 3 & 0.86 & $0.75-0.99$ & 0.04 & Fixed & 1.52 & 0.47 & $0 \%$ & & & & \\
\hline & Asian & 7 & 1.06 & $0.89-1.26$ & 0.52 & Fixed & 5.87 & 0.44 & $0 \%$ & & & & \\
\hline \multirow[t]{3}{*}{$G G \vee s . A G+A A$} & Overall & 10 & 0.88 & $0.67-1.17$ & 0.38 & Random & 14.23 & 0.11 & $37 \%$ & -0.80 & 0.42 & 1.89 & 0.10 \\
\hline & European & 3 & 0.97 & $0.79-1.20$ & 0.81 & Fixed & 0.85 & 0.65 & $0 \%$ & & & & \\
\hline & Asian & 7 & 0.65 & $0.36-1.19$ & 0.17 & Random & 12.58 & 0.05 & $52 \%$ & & & & \\
\hline \multirow[t]{3}{*}{ Pvull (C vs. T) } & Overall & 17 & 0.98 & $0.93-1.03$ & 0.37 & Fixed & 19.58 & 0.24 & $18 \%$ & 0.66 & 0.51 & 0.87 & 0.40 \\
\hline & European & 10 & 0.97 & $0.90-1.04$ & 0.14 & Random & 13.61 & 0.14 & $34 \%$ & & & & \\
\hline & Asian & 7 & 1.07 & $0.95-1.21$ & 0.25 & Fixed & 3.19 & 0.78 & $0 \%$ & & & & \\
\hline \multirow[t]{3}{*}{$C C$ vs. $T T+C T$} & Overall & 17 & 0.97 & $0.89-1.06$ & 0.55 & Fixed & 13.09 & 0.67 & $0 \%$ & 0.08 & 0.93 & 0.26 & 0.80 \\
\hline & European & 10 & 0.94 & $0.85-1.04$ & 0.21 & Fixed & 5.05 & 0.83 & $0 \%$ & & & & \\
\hline & Asian & 7 & 1.17 & $0.94-1.47$ & 0.16 & Fixed & 4.94 & 0.55 & $0 \%$ & & & & \\
\hline \multirow[t]{3}{*}{$C T$ vs. $C C+\Pi$} & Overall & 17 & 0.99 & $0.92-1.06$ & 0.75 & Fixed & 19.85 & 0.23 & $19 \%$ & 0.41 & 0.68 & 0.73 & 0.48 \\
\hline & European & 10 & 1.01 & $0.91-1.13$ & 0.82 & Random & 15.43 & 0.08 & $42 \%$ & & & & \\
\hline & Asian & 7 & 0.96 & $0.81-1.14$ & 0.64 & Fixed & 4.29 & 0.64 & $0 \%$ & & & & \\
\hline \multirow[t]{3}{*}{ TT vs. $\mathrm{CC}+\mathrm{CT}$} & Overall & 17 & 1.01 & $0.92-1.12$ & 0.79 & Random & 23.83 & 0.09 & $33 \%$ & -0.25 & 0.81 & -1.15 & 0.27 \\
\hline & European & 10 & 1.02 & $0.90-1.17$ & 0.74 & Random & 20.33 & 0.02 & $56 \%$ & & & & \\
\hline & Asian & 7 & 0.95 & $0.80-1.13$ & 0.57 & Fixed & 2.35 & 0.88 & $0 \%$ & & & & \\
\hline
\end{tabular}




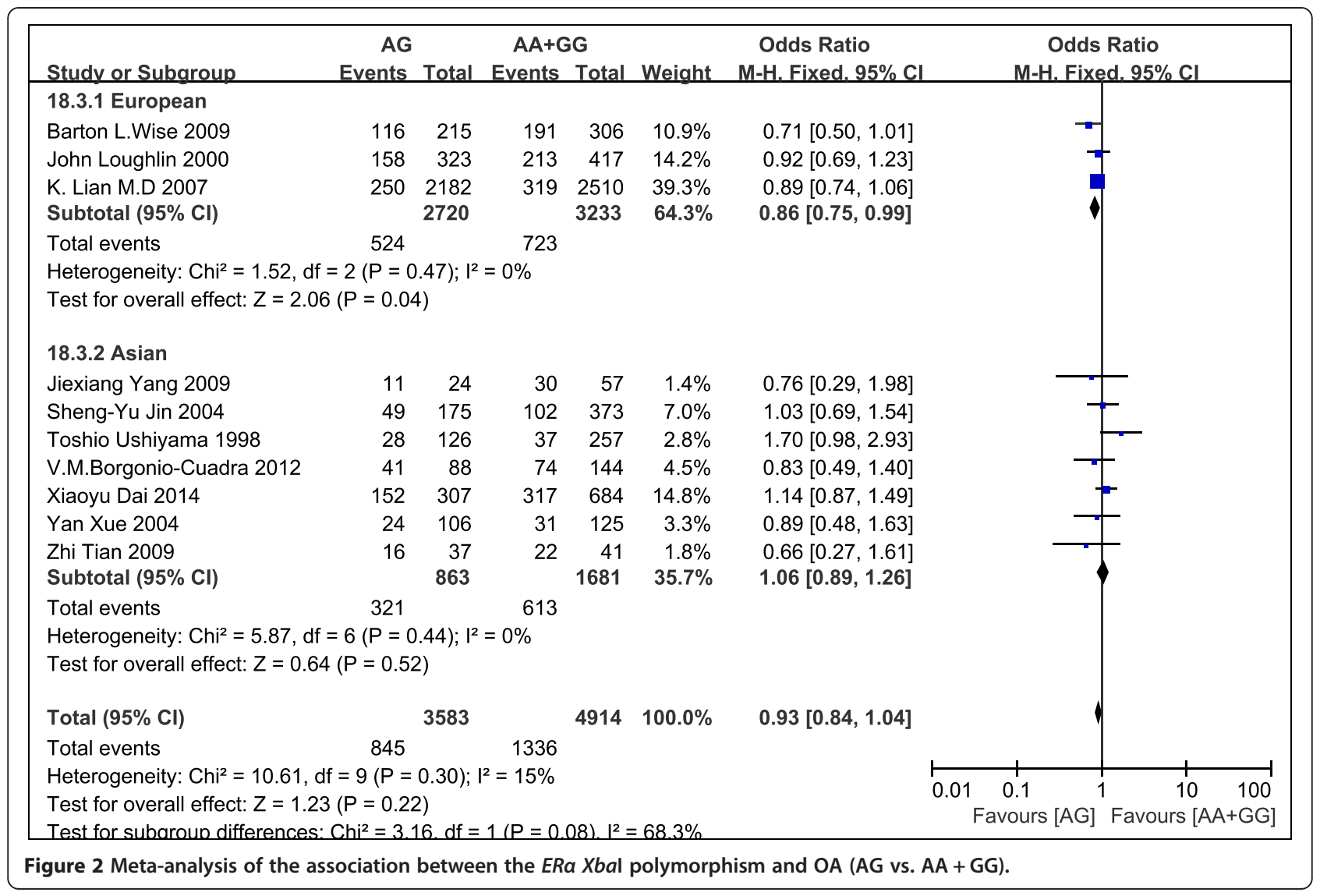

\section{Sensitivity analysis and publication bias}

As shown in Table 4, heterogeneity was observed among studies in all populations and also in subgroup analyses. To explore the sources of heterogeneity across studies we performed a sensitivity analysis, which revealed that none of the studies significantly affected the pooled ORs and CIs. Sequential removal of each study had little effect on the pooled ORs.

The funnel plot revealed no obvious publication bias (Figure 4), and this was confirmed by Begg's test and Egger's test.

\section{Discussion}

Although the pathogenesis of OA is considered to be the result of many factors, genetics are thought to be one of the most important determinants [42]. Despite the fact that ER $\alpha$ is one of the most studied genes in OA [43], to the best of our knowledge this is the first meta-analysis of the relationship between ER $\alpha$ polymorphisms XbaI and PvuII and OA risk.

Our meta-analysis included 11 published studies (with 17 comparisons) of 16,159 participants (5,325 OA patients and 10,834 controls). Ten studies with a total of 8,502 participants evaluated the association between the ER $\alpha$ XbaI polymorphism and OA susceptibility, and our meta-analysis suggested that it was significantly associated with $\mathrm{OA}$ in European but not Asian populations. The pooled OR for homozygote AA carriers showed that they were associated with a $17 \%$ increased risk for OA compared with AG and GG carriers, and that European AG carriers had a decreased OA risk. The heterogeneity of genetic effects between European and Asian populations suggests the existence of gene-environment or gene-gene interactions. No heterogeneity was detected in European populations with respect to the ER $\alpha \mathrm{XbaI}$ polymorphism and OA, suggesting that the genetic effect of this polymorphism is stronger in European than Asian populations.

Seventeen studies with a total of 16,159 participants evaluated the association between the ER $\alpha$ PvuII polymorphism and OA susceptibility. Our meta-analysis suggested that there was no association between the polymorphism and susceptibility to OA in any population. The same result was obtained for the subgroup analysis based on ethnicity.

Gender differences are known to affect the development of OA; for example, the prevalence of knee OA is greater in women than men [15]. Only two of the studies included in our meta-analysis were stratified according to participant gender [25,32], and both reported no significant 


\begin{tabular}{|c|c|c|c|c|c|c|c|}
\hline \multirow{2}{*}{ Study or Subgroup } & \multirow{2}{*}{$\begin{array}{r}\text { CC } \\
\text { Events }\end{array}$} & \multicolumn{3}{|c|}{$\mathrm{TT}+\mathrm{CT}$} & \multirow{2}{*}{ Weight } & \multirow{3}{*}{$\begin{array}{l}\text { Odds Ratio } \\
\text { M-H. Fixed. } 95 \% \mathrm{Cl}\end{array}$} & \multirow{2}{*}{$\begin{array}{l}\text { Odds Ratio } \\
\text { M-H. Fixed. } 95 \% \mathrm{Cl}\end{array}$} \\
\hline \multirow{2}{*}{\multicolumn{7}{|c|}{ 17.2.1 European }} & \\
\hline & & & & & & & \\
\hline Barton L.Wise 2009 & 58 & 104 & 246 & 411 & $4.6 \%$ & $0.85[0.55,1.31]$ & \\
\hline J.A. Riancho-1 hip 2010 & 50 & 99 & 202 & 397 & $4.1 \%$ & $0.99[0.63,1.53]$ & - \\
\hline J.A. Riancho-2 hip 2010 & 257 & 424 & 1021 & 1716 & $16.5 \%$ & $1.05[0.84,1.30]$ & - \\
\hline J.A. Riancho-2 knee 2010 & 77 & 244 & 368 & 1063 & $9.8 \%$ & $0.87[0.65,1.17]$ & + \\
\hline J.A. Riancho-3 hip 2010 & 80 & 259 & 279 & 902 & $8.9 \%$ & $1.00[0.74,1.35]$ & $t$ \\
\hline J.A. Riancho-3 knee 2010 & 53 & 232 & 219 & 842 & $7.6 \%$ & $0.84[0.60,1.19]$ & 7 \\
\hline J.A. Riancho-4 knee 2010 & 46 & 126 & 208 & 601 & $4.7 \%$ & $1.09[0.73,1.62]$ & $\sigma$ \\
\hline J.A. Riancho-4hip 2010 & 51 & 131 & 236 & 629 & $5.2 \%$ & $1.06[0.72,1.56]$ & 5 \\
\hline John Loughlin 2000 & 74 & 146 & 297 & 594 & $6.0 \%$ & $1.03[0.72,1.48]$ & 7 \\
\hline K. Lian M.D 2007 & 102 & 986 & 465 & 3694 & $18.2 \%$ & $0.80[0.64,1.01]$ & \#+ \\
\hline Subtotal $(95 \% \mathrm{Cl})$ & & 2751 & & 10849 & $85.6 \%$ & $0.94[0.85,1.04]$ & 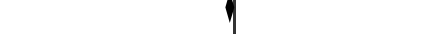 \\
\hline Total events & 848 & & 3541 & & & & \\
\hline \multicolumn{8}{|c|}{ Heterogeneity: $\mathrm{Chi}^{2}=5.05, \mathrm{df}=9(\mathrm{P}=0.83) ; \mathrm{I}^{2}=0 \%$} \\
\hline \multicolumn{8}{|c|}{ Test for overall effect: $Z=1.25(P=0.21)$} \\
\hline \multicolumn{8}{|l|}{ 17.2.2 Asian } \\
\hline Jiexiang Yang 2009 & 10 & 15 & 31 & 66 & $0.4 \%$ & $2.26[0.70,7.33]$ & \\
\hline Sheng-Yu Jin 2004 & 22 & 84 & 129 & 464 & $3.0 \%$ & $0.92[0.54,1.56]$ & - \\
\hline Toshio Ushiyama 1998 & 11 & 68 & 54 & 315 & $1.7 \%$ & $0.93[0.46,1.90]$ & - \\
\hline V.M.Borgonio-Cuadra 2012 & 14 & 30 & 101 & 202 & $1.4 \%$ & $0.88[0.41,1.89]$ & - \\
\hline Xiaoyu Dai 2014 & 85 & 159 & 384 & 824 & $6.0 \%$ & $1.32[0.94,1.85]$ & - \\
\hline Yan Xue 2004 & 15 & 47 & 40 & 184 & $1.1 \%$ & $1.69[0.83,3.42]$ & \\
\hline Zhi Tian 2009 & 7 & 16 & 31 & 62 & $0.7 \%$ & $0.78[0.26,2.35]$ & 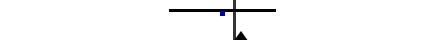 \\
\hline Subtotal $(95 \% \mathrm{CI})$ & & 419 & & 2117 & $14.4 \%$ & $1.17[0.94,1.47]$ & 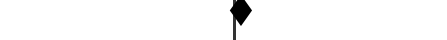 \\
\hline Total events & 164 & & 770 & & & & \\
\hline \multirow{2}{*}{\multicolumn{8}{|c|}{$\begin{array}{l}\text { Heterogeneity: } \text { Chi }^{2}=4.94, d f=6(P=0.55) ; I^{2}=0 \% \\
\text { Test for overall effect: } Z=1.39(P=0.16)\end{array}$}} \\
\hline & & & & & & & \\
\hline Total $(95 \% \mathrm{Cl})$ & & 3170 & & 12966 & $100.0 \%$ & $0.97[0.89,1.06]$ & $f$ \\
\hline Total events & 1012 & & 4311 & & & & \\
\hline Heterogeneity: $\mathrm{Chi}^{2}=13.09$ & $f=16(P$ & $=0.67)$ & $I^{2}=0 \%$ & & & & $0.01 \quad 0.1$ \\
\hline $\begin{array}{l}\text { Test for overall effect: } Z=0 \text {. } \\
\text { Test for subaroun difference }\end{array}$ & $\begin{array}{l}(P=0.5 \\
C h i^{2}=3\end{array}$ & 5) $14 \mathrm{df}=$ & $(P=0$ & 8) $1^{2}=$ & $681 \%$ & & Favours $[\mathrm{CC}]$ Favours $[\mathrm{TT}+\mathrm{CT}]$ \\
\hline
\end{tabular}

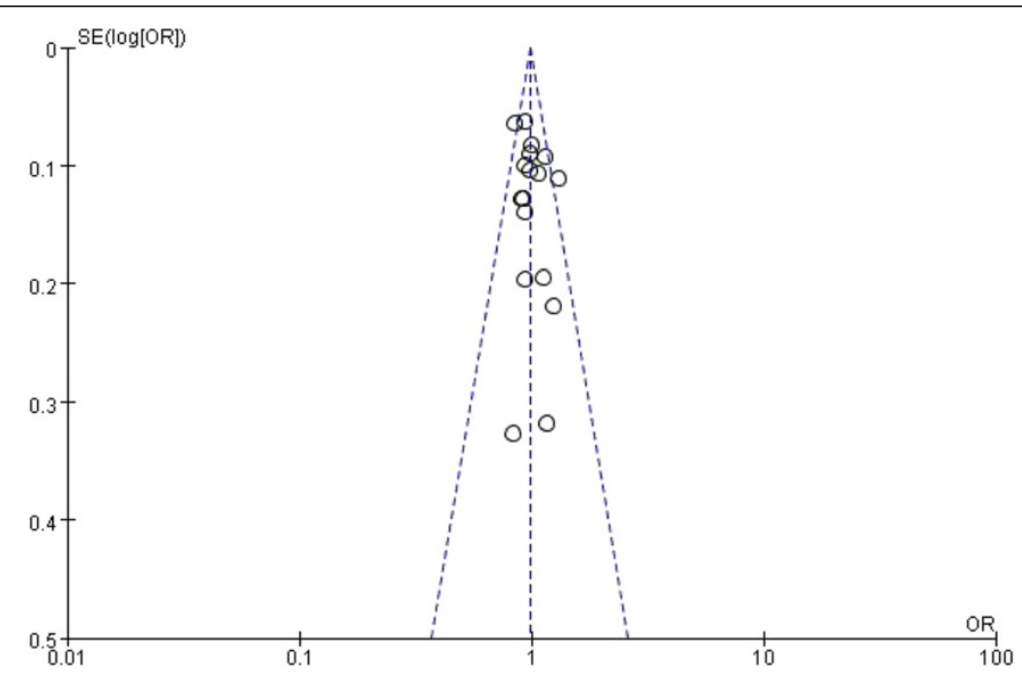

Figure 4 Funnel plot of the meta-analysis of the ERa Pvull polymorphism with susceptibility to OA (C vs. T). 
differences in the $E R \alpha$ polymorphisms between OA patients and controls of the same sex. However, because of the small number of this type of study and the limited raw data based on gender differences in genotype distributions and allele frequencies, we were unable to perform a subgroup analysis according to gender.

Several limitations should be taken into consideration in the current meta-analysis. First, it was based on unadjusted OR estimates because not all studies presented adjusted ORs, or the ORs were not adjusted by the same potential confounders, such as age and gender. This lack of information could have caused serious confounding bias. Second, OA is influenced by both genetic and environmental risk factors such as obesity, injury, occupational activities, and meniscectomy. However, the studies included in the meta-analysis did not control for these environmental risk factors. Third, some studies included individuals with $\mathrm{OA}$ in different sites, but we were unable to perform subgroup analysis of this within the same ethnic population because of the limited available data. For instance, hand OA is known to be more influenced by genetic and hormonal influences than other types of OA, but the relationship between the ER $\alpha \mathrm{Xba \textrm {I }}$ polymorphism and hand OA was only reviewed in one study of Europeans [30] and one of Asians [24]. Other studies of the ER $\mathrm{XbaI}$ polymorphism and OA susceptibility in Europeans focused on three different OA sites. Finally, although our current findings suggest that the $E R \alpha X b a \mathrm{I}$ polymorphism is associated with $\mathrm{OA}$ in Europeans, it was not possible to determine whether this polymorphism is in linkage disequilibrium with any other potentially functional polymorphisms. However, our meta-analysis also had some advantages, including a satisfactory quality of all 11 included studies, and a well-designed method.

\section{Conclusions}

The present results suggest that there may be a weak relationship between the $E R \alpha X b a \mathrm{I}$ polymorphism and $\mathrm{OA}$ in European but not Asian populations, while the $E R \alpha$ $P v u I I$ polymorphism did not appear to be associated with OA in either Europeans or Asians. Because the studies included in the meta-analysis reviewed the relationship between the ER $\alpha \mathrm{Xba \textrm {I }}$ polymorphism and OA susceptibility at three different sites in Europeans, large well-designed studies are necessary to confirm our findings in more homogeneous populations.

\section{Competing interests}

The authors declare that they have no competing interests.

\section{Authors' contributions}

$Y R, B T, Y W u$, and $Y$ Wang participated in the conception and design of the study. YR and BT carried out the literature search. YR, BT, and YY carried out the data collection. YR, BT, and PY performed the statistical analysis. Y Wu and $Y$ Wang assessed the quality of the studies. YR and BT wrote the manuscript. $Y$ Wu and $Y$ Wang revised the manuscript. All authors read and approved the final manuscript.

\section{Acknowledgment}

This study was supported by grants from the Doctor Foundation of Sichuan Provincial People's Hospital (30305030575). The authors thank Professor Deiying Kang for advice on modifying the meta-analysis, Min Yang for advice on data analysis, and Bangliang Xiao for logistics support.

\section{Author details}

'Department of Epidemiology and Biostatistics, West China School of Public Health, Sichuan University, Chengdu 610041, People's Republic of China.

${ }^{2}$ Department of Orthopedics, Sichuan Academy of Medical Sciences, Sichuan Provincial People's Hospital, Chengdu 610072, People's Republic of China. ${ }^{3}$ Department of Preventive Health Care, The People's Hospital of Dazu District, Chongqing 402360, People's Republic of China. ${ }^{4}$ Department of Medical Record Management, West China Second University Hospital, Sichuan University, Chengdu 610041, People's Republic of China.

Received: 16 September 2014 Accepted: 19 February 2015

Published online: 27 February 2015

\section{References}

1. Felson DT, Zhang Y. An update on the epidemiology of knee and hip osteoarthritis with a view to prevention. Arthritis Rheum. 1998:41:1343-55.

2. Felson DT. Developments in the clinical understanding of osteoarthritis. Arthritis Res Ther. 2009;11(1):203-14.

3. Chapman K, Takahashi A, Meulenbelt I, Watson C, Rodriguez-Lopez J, Egli R, et al. A meta-analysis of European and Asian cohorts reveals a global role of a functional SNP in the $5^{\prime} U T$ R of GDF5 with osteoarthrit is susceptibilit $y$. Hum Mol Genet. 2008;17(10):1497-504.

4. Nakamura T, Shi DQ, Tzetis M, Rodriguez-Lopez J, Miyamoto Y, Tsezou A, et al. Meta-analysis of association between the ASPN D-repeat and osteoarthritis. Hum Mol Genet. 2007;16(14):1676-81.

5. Valdes AM, Loughlin J, Oene MV, Chapman K, Surdulescu GL, Doherty M, et al. Sex and ethnic differences in the association of ASPN, CALM 1 , COL2A1, COMP, and FRZB with genetic susceptibility to osteoarthritis of the knee. Arthritis Rheum. 2007:56(1):137-46.

6. Gálvez-Rosas A, González-Huerta C, Borgonio-Cuadra VM. A COL2A1 gene polymorphism is related with advanced stages of osteoarthrit is of the knee in Mexican Mestizo population. Rheumatol Int. 2010;30(8):1035-9.

7. Jiang $L Y$, Tian WJ, Wang YC, Rong JS, Bao CD, Liu YP, et al. Body mass index and susceptibility to knee osteoarthritis: A systematic review and meta-analysis. Joint Bone Spine. 2012;79:291-7.

8. Lee R, Kean WF. Obesity and knee osteoarthritis. Inflammopharmacol. 2012;20:53-8.

9. Jiang $L Y$, Rong JS, Wang YC, Hu F, Bao CD, Li X, et al. The relationship between body mass index and hip osteoarthritis: A systematic review and meta-analysis. Joint Bone Spine. 2011;78:150-5.

10. Muthuri SG, McWilliams DF, Doherty M, Zhang W. History of knee injuries and knee osteoarthritis: a meta-analysis of observational Studies. Osteoarthr Cartil. 2011;19:1286-93.

11. McWilliams DF, Leeb BF, Muthuri SG, Doherty M, Zhang W. Occupational risk factors for osteoarthritis of the knee: a meta-analysis. Osteoarthr Cartil. 2011;19:829-39.

12. Palmer KT. Occupational activities and osteoarthritis of the knee. Br Med Bull. 2012;102:147-70.

13. Tanamas SK, Wijethilake P, Wluka AE, Davies-Tuck ML, Urquhart DM, Wang $Y$, et al. Sex hormones and structural changes in osteoarthritis: A systematic review. Maturitas. 2011;69:141-56.

14. Papalia R, Buono AD, Osti L, Denaro V, Maffulli N. Meniscectomy as a risk factor for knee osteoarthritis: a systematic review. Br Med Bull. 2011:99:89-106.

15. Blagojevicy M, Jinksy C, Jefferyz A, Jordany KP. Risk factors for onset of osteoarthritis of the knee in older adults: a systematic review and meta-analysis. Osteoarthr Cartil. 2010;18:24-33.

16. Loughlin J. Genetic epidemiology of primary osteoarthritis. Rheumatology. 2001;13:111-6.

17. Loughlin J. Familial Inheritance of Osteoarthritis. Lippincott Williams Wilkins. 2004;427S:S22-5. 
18. Tsai CL, Liu TK. Osteoarthritis in women:its relationship to estrogen and current trends. Life Sci. 1992;50(23):1737-44.

19. Richette P, Corvol M, Bardin T. Estrogens, cartilage, and osteoarthritis. Joint Bone Spine. 2003;70:257-62.

20. Cheung E, Schwabish MA, Kraus WL. Chromatin exposes intrinsic differences in the transcriptional activities of estrogen receptorsaand $\beta$. EMBO J. 2003;22:600-11.

21. Ponglikitmongkol M, Green S, Chambon P. Genomic organization of the human oestrogen receptor gene. EMBO J. 1988;7:3385-8.

22. Wang KJ, Shi DQ, Sun LS, Jiang X, Lü YY, Dai J, et al. Association of estrogen receptor alpha gene polymorphisms with bone mineral density: a meta-analysis. Chin Med J. 2012;125(14):2589-97.

23. Bergink AP, van Meurs JB, Loughlin J, Arp PP, Fang Y, Hofman A, et al. Estrogen receptor alpha gene haplotype is associated with radiographic osteoarthritis of the knee in elderly men and women. Arthritis Rheum. 2003;48(7):1913-22.

24. Ushiyama T, Ueyama H, Inoue K, Nishioka J, Ohkubo I, Hukuda S. Estrogen receptor gene polymorphism and generalized osteoarthritis. J Rheumatol. 1998;25:134-7.

25. Jin SY, Hong SJ, Yang HI, Park SD, Yoo MC, Lee HJ, et al. Estrogen receptors-a gene haplotypes is associated with primary knee osteoarthritis in Korean population. Arthritis Res Ther. 2004;6(5):415-21.

26. Xiaoyu Dai,Chao Wang, Jin Dai, Dongquan Shi, Zhihong Xu, Dongyang Chen, et al. Association of Single Nucleotide Polymorphisms in Estrogen Receptor Alpha Gene with Susceptibility to Knee Osteoarthritis: A Case-control Study in a Chinese Han Population. BioMed Res Int. 2014 (ID 151457):1-12. http://dx.doi.org/10.1155/2014/151457.

27. Xue Y, Li D, Li Y, Zhou YX, Xiao D, Guo SQ, et al. Relationship between Estrogen receptor gene polymorphism and osteoarthritis in Han women. Chin J Rheumatol. 2004:8:583-6.

28. Tian Z, Guo X, Zhou F, Huang CX, Su M, Long XL, et al. Study on Relationship Between Polymorphisms of Estrogena Receptor Gene and Primary Knee Osteoarthritis Among Women in Hunan. Pract Prev Med. 2009;6:1724-7

29. Loughlin J, Sinsheimer JS, Mustafa Z, Carr AJ, Clipsham K, Bloomfield VA, et al. Association analysis of the vitamin $\mathrm{D}$ receptor gene, the type I collagen gene COL1A1, and the estrogen receptor gene in idiopathic osteoarthritis. J Rheumatol. 2000:27:779-84.

30. Wise BL, Demissie S, Cupples LA, Felson DT, Yang M, Shearman AM, et al. The relationship of estrogen receptor-aand-Bgenes with osteoarthritis of the hand. J Rheumatol. 2009;36:2772-9.

31. Lian K, Lui L, Zmuda JM, Nevitt MC, Hochberg MC, Lee JM, et al. Estrogen receptor alpha genotype is associated with a reduced prevalence of radiographic hip osteoarthritis in elderly Caucasian women. Osteoarthr Cartil. 2007;15:972-8.

32. Verónica Marusa BC, Celia GH, Carolina DS, María SB, Socorro CG, Antonio $\mathrm{MD}$. Analysis of estrogen receptor alpha gene haplotype in Mexican mestizo patients with primary osteoarthritis of the knee. Rheumatol Int. 2012;32:1425-30.

33. Riancho JA, García-lbarbia C, Gravani A, Raine EV, Rodríguez-Fontenla C, Soto-Hermida A, et al. Common variations in estrogen-related genes are associated with severe large-joint osteoarthritis: a multicenter genetic and functional study. Osteoarthr Cartil. 2010;18:927-33.

34. Yang J, Shijie F, Xiao F-s. Case-control Study between Estrogen Receptor Gene Polymorphisms and Osteoarthritis in Southern Sichuan High Fluoride Areas. West China Med J. 2009;24(4):826-9.

35. Liberati A, Altman DG, Tetzlaff J, Mulrow C, Gøtzsche PC, loannidis JPA, et al. The PRISMA statement for reporting systematic reviews and meta-analyses of studies that evaluate healthcare interventions: explanation and elaboration. Ann Intern Med. 2009;151:W-65-94.

36. Stang A. Critical evaluation of the Newcastle-Ottawa scale for the assessment of the quality of nonrandomized studies in meta-analyses. Eur J Epidemiol. 2010;25(9):603-5.

37. Robins J, Greenland S, Breslow NE. A general estimator for the variance of the Mantele-Haenszel odds ratio. Am J Epidemiol. 1986;124:719-23.

38. Der Simonian R, Laird N. Meta-analysis in clinical trials. Control Clin Trials. 1986;:177-88

39. Egger M, Smith GD, Phillips AN. Meta-analysis: principles and procedures. BMJ. 1997;315:1533-7.
40. Duval S, Tweedie R. Trim and fill: A simple funnel-plot-based method of testing and adjusting for publication bias in meta-analysis. Biometrics. 2000;56:455-63.

41. Peters JL, Sutton AJ, Jones DR, Abrams KR, Rushton L. Performance of the trim and fill method in the presence of publication bias and between-study heterogeneity. Stat Med. 2007;26:4544-62.

42. Chapman K, Valdes AM. Genetic factors in OA pathogenesis. Bone. 2012;51:258-64.

43. Spector TD, MacGregor AJ. Risk factors for osteoarthritis: genetics. Osteoarthr Cartil. 2004;12:S39-44.

\section{Submit your next manuscript to BioMed Central and take full advantage of:}

- Convenient online submission

- Thorough peer review

- No space constraints or color figure charges

- Immediate publication on acceptance

- Inclusion in PubMed, CAS, Scopus and Google Scholar

- Research which is freely available for redistribution

Submit your manuscript at www.biomedcentral.com/submit

C Biomed Central 